\title{
A Novel Multiuser Detection and Channel Estimation Method for Uplink Communication in MIMO-OFDM IDMA System
}

\author{
Dolly Sharma,. Rabindra Kumar Singh, Himanshu Katiyar
}

\begin{abstract}
Demand of wireless cellular communication systems has grown rapidly which necessitates high speed and reliable communication for various multimedia applications. Several technologies have been introduced to meet the desired communication requirements such as $1 G, 2 G, 3 G$ and $4 G$ but these techniques suffer from multiple-access and interference issues. Hence in this work, we focus on these challenges to improve the cellular communication performance. The main objective of this work is to develop a novel approach for multiuser detection and introducing a new architecture of channel estimation. However, several techniques are present to achieve these objectives but computational complexity and reliable performance still remains an issue. Moreover, interference remains a challenging task. Hence, in order to overcome these issues, we present a joint approach of multiuser detection where we compute the LLRs, find the Gaussian distribution and measure the mean and variance of this distribution. Similarly, in next phase, channel estimation is performed with the help of ESE and DEC. The performance of proposed approach is measured in terms of BER for varied simulation configurations. The experimental analysis reported significant improvement in the performance of MIMO-OFDM IDMA system.
\end{abstract}

\section{INTRODUCTION}

During last two decade, we have noticed significant growth in wireless cellular communication and technologies. The next generation wireless communication demand for high speed and reliable communication to support various multimedia applications. However, multiple users communicate in the same environment where it becomes a challenging issue to deliver the reliable communication due to interference and multi-path fading [1]. Several wireless cellular communication standards are present such as $1 \mathrm{G}, 2 \mathrm{G}$, 3G and 4G [2]. Moreover, now-a-days, LTE (Long Term Evaluation) and LTE-A (Long Term Evaluation-Advanced) techniques [3] have been introduced to satisfy the demand of high data rate communication. Incorporating multiple access (MA) techniques in these communication models is considered as a promising solution to improve the performance. Currently, several multiple access schemes are present such as time-division multiple access (TDMA) [3], Frequency-Division Multiple Access (FDMA) [4],

Revised Manuscript Received on October 22, 2019.

* Correspondence Author

Dolly Sharma*, Assistant Professor, EC Department, BBDEC, Lucknow Dr. Rabindra Kumar Singh, Former Professor, Electronics Engineering, KNIT, Sultanpur Sonbhadra

E.Mail

id:

phd.dolly.sharma@gmail.com,

singhrabinder57@gmail.com, katiyarhimanshu@gmail.com
Dr. Himanshu Katiyar, Associate Professor, Electronics Engineering, REC

Code-Division Multiple access (CDMA) [5], orthogonal frequency-division multiple access (OFDMA) [6] , non-orthogonal frequency-division multiple access (NOMA) [7] and Interleave Division Multiple Access (IDMA) [8].

The IDMA systems are considered as a promising technique to improve the communication performance. The IDMA based models are used in MIMO [9], NOMA [10], and OFDM [11] etc. The IDMA systems have significant impact on $5 \mathrm{G}$ communication where huge numbers of users are present. The IDMA takes advantage of its own chip-level interleaver to separate the multiple users at the receiver. The IDMA receiver contains the elementary signal estimator (ESE) which is based on the soft rake processing approach and performs the chip level detection at the receiver end. Once the ESE task is finished, the detected chip LLRs are processed through de-interleaving and de-spreading to obtain the bit level LLRs. Later, the obtained LLRs are given as input to the channel decoder. Moreover, the de-interleaver and data despreading process is considered as a channel decoding process which helps to minimize the error. Currently, IDMA has gained attraction because it can be applied in various communication systems such as multi-carrier transmission, MIMO transmission, joint detection and channel estimation schemes. In [12] IDMA systems are implemented with multiple access and non-Gaussian noise. Bilim et al. [13] reported that the IDMA achieves good performance with flat fading and additive white noise. However, these systems achieve poor performance for multi-path fading scenario because multiple paths reduce the convergence capability of ESE. In order to overcome this issue multiuser detection schemes are developed [11] [14-17].

Compared to CDMA, multiuser detection algorithms are easy to implement in IDMA systems. However, the convergence speed of these approaches becomes a tedious task which depends on the signal to interference and noise ratio (SINR) Generally, value of SINR is low during initial iterations but increases slowly, thus, the numbers of iterations required for computation are more in IDMA systems. On other hand, the computational complexity at the receiver end is a challenging issue. According to the conventional architecture of IDM, it has to detect all the signal of other available users for further iterative receiving process at user end. In this context, the channel estimation techniques are implemented [18-20]. The computational complexity for multiple users is a key issue in these types of models. On the other hand, the interleavers are used for distinguishing the signals from different users but it is not convenient for one user to know all parameters of other user's interleaving parameters.

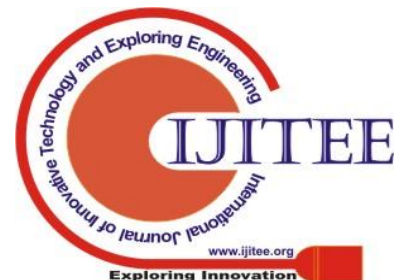




\section{A Novel Multiuser Detection and Channel Estimation Method for Uplink Communication in MIMO-OFDM IDMA System}

Moreover, the IDMA systems suffer from the co-channel and inter-symbol interference which leads to degraded performance for uplink and downlink communication in IDMA system. Currently, cooperative schemes are widely studied in these applications such as Bilim et al. [21] cooperative decode and Forward cooperative scheme using best relay selection. In another study, presented in [22], authors introduced cooperative relay beamforming scheme in IDMA. Similarly, Vishvaksenan et al. [23] presented relay aided cooperative downlink communication approach.

In this work, we focus on the uplink communication and developed a novel approach for uplink communication along with channel coding and interleaving. The main contribution of this work is as follows:

(a) Development of an uplink IDMA system

(b) Presenting the chip-by chip detection model for multi-path uplink communication.

(c) Development of channel coding and interleaving scheme to mitigate the error rate.

Rest of the manuscript is organized in following sub-sections: section II presents brief discussion on current literature in the field of IDMA systems. Section III presents proposed solution to improve the overall performance. Section IV presents experimental and comparative analysis using proposed approach and finally, section $\mathrm{V}$ gives concluding remarks.

\section{LITERATURE SURVEY}

This section presents a brief discussion about recent techniques to improve the performance of IDMA systems. Multiuser detection, beamforming, cooperative communication and relay selection etc. are the significant research components of IDMA.

Sharma et al. [11] introduced an approach for multi-user detection and interference cancellation in IDMA. According to this approach spatial log-likelihood multiuser detection is carried out and tested for MIMO-IDMA systems. Moreover, authors have developed modulation sub-carrier classification approximation model.

$\mathrm{Li}$ et al. [14] discussed that the conventional $\mathrm{CBC}$ (Chip-by-Chip) detectors consider that the sensor activity is precisely known. However, this is not convenient for all receivers hence, authors proposed a multiuser detection approach in sporadic IDMA for uplink transmission. In order to achieve this, compressed sensing is applied for MUD activity analysis and data detection. Later, compressed sensing and $\mathrm{CBC}$ are combined and CS-CBC detector is developed. Similar to this, Shikida et al. [24] introduced a combined multiuser detection and channel estimation approach for uplink MIMO-OFDM IDMA communication. The main concept of this work is based on the iterative receiver where MUD and channel estimation techniques are performed jointly. This is achieved by computing log-likelihood ratios of coded bits (bit LLRs). During this process, soft decision-directed channel estimation (SDCE) is applied where bit LLRs are used along with the initial channel estimation. Later, tap selection process is presented to improve the channel estimation. Tap selection is used to extract the dominant paths.

Several techniques have been reported for channel estimation in OFDM-IDMA systems. TAşPINAR et al. [25] presented Least Squares (LS) and Minimum Mean Square Error
(MMSE) based MUD approach to estimate the channel frequency response. Peng et al. [26] discussed that the uplink OFDM-IDMA systems suffer from the carrier frequency offsets. In order to overcome this issue, authors suggested an approach for iterative estimation and cancellation of addition interference. Chen et al. [27] introduced iterative interference cancellation multiuser detection (IIC-MUD) approach for Overloaded MIMO OFDM IDMA System. In [19] Chang et al. presented a combined approach to time tracking and channel estimation for IDMA. In this work, authors have implemented pilot-assisted turbo-type iterative detection model which can be used for recovering the message of each user from the obtained superimposed signals.

On other hand, cooperative schemes are also widely studied in this field. Generally, cooperative schemes are based on the concept of relay selection where two schemes decode -forward (DF) and amplify -forward (AF) are used. Zhou et al. [28] presented superposition modulated cooperative scheme for multiple source node scenario. According to this approach, the source nodes are divided into several cooperative subsets in a Time Division Duplexing (TDD) manner. During transmission, the signal is superimposed which contains its own data information and data obtained from the previous subset node. The node subsets are differentiated using IDMA to receive the signal. Yang et al. [29] presented network coding concept in IDMA and introduced a cooperative relay selection scheme. This work uses Turbo coding model for multi-path systems. Moreover, this approach adopts different interleaver for different users and also network coding cooperative schemes are studied. The cooperative approach is used for beamforming in IDMA where optimal relay selection is carried out. Shukla et al. [22] focused on beamforming and relay selection in IDMA. In this work, IDMA and cooperative relay aided beamforming is developed. This approach is divided into two phases where first of all, users broadcast the message, the relay nodes perform the amplification task and forwards the data to the destination node. In order to mitigate the effect of multiple access interference (MAI), the IDMA scheme is incorporated at relay and destination nodes. This can be achieved using efficient beamforming. Hence, authors presented a beam forming approach to minimize the transmit power and controls the QoS parameters. Moreover, the power minimization is not considered as convex optimization problem hence semi-definite problem is formulated and solved using CVX solver.

Similarly, researchers have presented error correcting codes for minimizing the bit error rates and improve the QoS performance. Zhang et al. [30] focused on power and linear density parity check (LDPC) model optimization to improve the IDMA performance. In order to achieve this, the power profiling is considered as main task which are obtained using mutual information between users. Further, a Gaussian approximation method is applied to compute the probability density function. According to the receiver architecture, the detector and decoder exchange the information in an iterative process.

Zhang et al. [31] developed raptor-like quasi-cyclic low density parity check (RL-QC-LDPC) to improve the IDMA system for $5 \mathrm{G}$ communication applications. 
The conventional signal estimator processes data symbol-by-symbol manner whereas in this process, the signal estimator is replaced by a newly designed maximum a posterior probability multi-user detection.

This section presents a brief discussion about recent techniques of multiple access using IDMA. Several new architectures are discussed along with new interleaver designs to improve the communication performance. Moreover, IDMA schemes are also incorporated with MIMO OFDM systems to satisfy the communication requirement. In addition to this, several researchers have presented forward error correcting techniques to reduce the packet error. However, due to increasing complexity and demand, these systems fail to achieve the desired performance in terms of bit error rate and complexity. Hence, there is a need to present a novel approach to meet the performance requirement.

\section{PROPOSED MODEL}

This section presents the proposed solution to improve the performance of IDMA systems. In order to achieve this, we divide the proposed solution into two phases as (a) low-complexity multi-user detection and (b) channel estimation. The proposed model provides a joint solution for MUD and channel estimation.

\section{(a)Multiuser detection}

$$
\operatorname{Var}\left[\bar{\eta}_{k l}(n)\right]
$$

Let us consider an IDMA system with $K$ users as depicted in figure 1 which has recursive systematic convolutional (RSC) code model (rate of RSC is $R=\frac{1}{2}$ ) to protect the $b_{k}(n)$ information bits of $k$ user. The obtained coded bits are permuted using interleavers $I_{k}$ and modulated using QPSK modulation scheme. The current baseband signal can be expressed as:

$$
r(t)=\sum_{i=0}^{L-1} h_{l}(t) y\left(t-\tau_{l}\right) e^{j \phi_{l}(t)}+w(t)
$$

where $y(t)$ denotes the transmitted signal expressed as $\left[t_{\gamma}(t) \sum_{k=1}^{K} x_{k}(t)\right]$ where $t_{\gamma}$ denotes the Binary Phase Shift Keying (BPSK) modulated training sequence, at this stage signals of all users are combined, $h_{l}(t)$ denotes the fading coefficient for path $l$ at time $t$, the total path delay is $\tau_{\mathbb{l}}$, total number of path $L$ and phase $\phi, w(t)$ denotes the additive

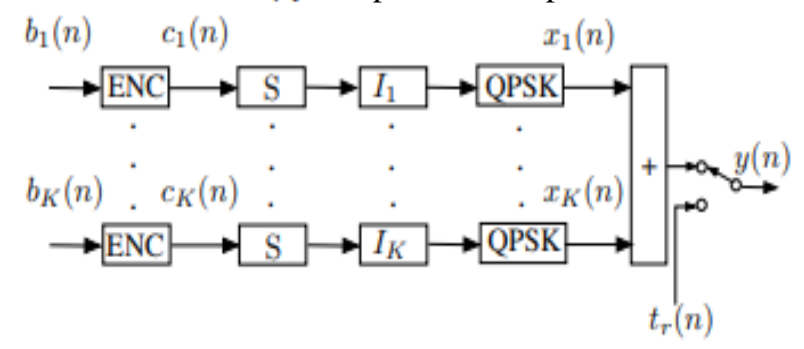

Recently, interference cancellation and multiuser detection techniques are adopted to mitigate the effect of interference, MAI and MUD [11]. According to these schemes, detector considers the multipath signal and detects each signal individually by computing log-likelihood ratios (LLRs). These LLRs are aggregated as $(L+1)$ LLRs for each chip which helps to achieve the more accurate information of signal. After processing through MUD models, the interference of $k$ users $\eta_{\left.k_{i}\right]}(n)$ can be represented as Gaussian random variable which can be expressed as: Gaussian noise, and $\phi_{l}(t)$ represents the phase distortion.

$$
\bar{r}(n+1)=h_{k_{l} l}^{*}(n+l)=\left|h_{k_{l} l}^{*}(n)\right|^{2} x_{k}(n)+\bar{\eta}_{k_{l} l}(n)
$$

7. (nil) denotes the remaining interference of MUD models. From above expression, the outcome of detector can be approximated with the help of Gaussian distribution. Here, we compute the mean and variance of the baseband signal as:

$$
E[r(n)]=\sum_{k_{l} l} h_{k_{l} l}(n) E\left[x_{k}(n-l)\right]_{,} \forall_{n}
$$

To compute the variance, we consider the total number of bits and the data bits in physical propagation path. The difference of bit is computed and mean value is obtained for baseband signal. Similar task is performed in eq.(5) where we compute the mean and variance for interference signal.

Similarly, the covariance of this baseband signal is computed

$\operatorname{Cov}[\boldsymbol{r}(n)]=\sum_{k_{d} l} H_{k_{l} l}(n) \operatorname{Cov}\left[x_{k}(n-l)\right] H_{k_{l} I}^{T}(n)+\sigma^{2} A_{s} \forall_{n}$

where $\sigma$ is the noise variance and $A$ representes the $2 \times 2$

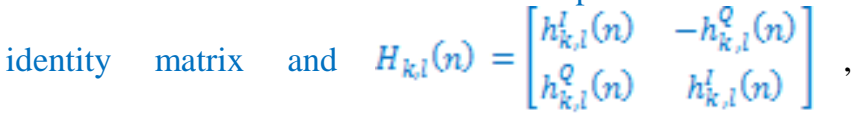

Similarly, the mean and variance of interference is computed as given in Eq. (5) and (6)

$E\left[\bar{\eta}_{k_{l} l}(n)\right]=h_{k l}^{*}(n) E[r(n+l)]-\left|h_{k_{l} l}(n)\right|^{2} E \mid x_{k}(n) \|_{,} \forall k_{s} n$ Variance of this interference can be computed as:

$=H_{k_{l} l}^{T}(n)\left[\operatorname{Cov}[\boldsymbol{r}(n+l)]-H_{k_{l} l}(n) \operatorname{Cov}\left[\left[\boldsymbol{x}_{k}(n)\right] H_{k_{l} l}^{T}(n)\right] H_{k_{l} l}(n), \forall k_{s}, n\right.$

In the next phase, we generate the LLRs and combine them to detect the multi user signals. This is expressed as:

$L_{M U D}\left[x_{k}^{I}(n)\right]_{L}=2\left|h_{k_{l} l}(n)\right|^{2} \frac{\bar{r}^{I}(n+l)-E\left[\eta_{k l}^{-I}(n)\right]}{\operatorname{Var}\left[\zeta_{k_{l} l}^{-I}(n)\right]}, \forall k_{,} n$ $\bar{F}^{l}$ denotes the real part of the received signal. For multiple paths, it can be rewritten as:

$$
L_{M U D}\left[x_{k}^{I}(n)\right]_{L}=\sum_{l=0}^{L-1} L_{M U D}\left[x_{k}^{I}(n)\right]_{L}, \forall k, n
$$

In order to obtain the accurate information, the priori means and signal variances of the transmitted signal are required which are computed using Eq (6) with the help of extrinsic LLRs of the decoder, given as:

$$
\begin{aligned}
& E\left[x_{k}(n)\right]=\tanh \left(\frac{L_{d e c}\left[x_{k}^{I}(n)\right]}{2}\right)+j \tanh \left(\frac{L_{d e c}\left[x_{k}^{Q}(n)\right]}{2}\right) \\
& \text { and } \\
& \operatorname{Cov}\left[x_{k}(n)\right]=\left[\begin{array}{cc}
1-E\left[x_{k}^{I}(n)\right]^{2} & 0 \\
0 & 1-E\left[x_{k}^{Q}(n)\right]^{2}
\end{array}\right]
\end{aligned}
$$

Here, $x_{k}^{T}$ and $x_{k}^{Q}$ are uncorrelated real and imaginary part of the $x(n), E[$ ] And $\operatorname{Vor}[\mathrm{r}]$ denotes the mean and variance of the signal. This approach can be extended for multiple path to perform the MUD.

\section{(b) Channel Estimation}

In this section, we present the proposed solution for channel estimation for IDMA systems. In this stage, we consider that the receiver module has an idle channel state information. Figure 2 shows an architecture of proposed channel estimator. 


\section{A Novel Multiuser Detection and Channel Estimation Method for Uplink Communication in MIMO-OFDM IDMA System}

In order to estimate the channel, we present pilot embedding model for a synchronous channel without memory. For simplicity, we rewrite Eq. (1) as:

$$
r=\sum_{l=1}^{K} h_{k} x_{k}+n
$$

Let $P$ be the pilot for $k$ user denoted as $p_{k} \equiv\left[p_{k}(1), \ldots p_{k}(j)\right]$, here, we assume that the $\left\{p_{k}\right\}$ are generated randomly and remain independent.

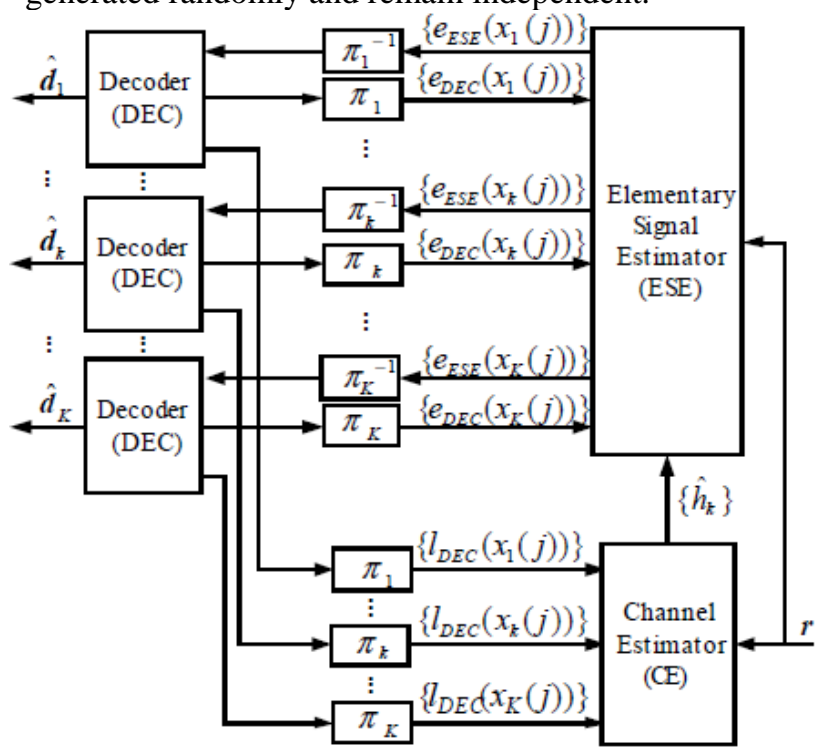

Fig.2. Channel estimator

After considering the transmitted signal from $k^{\text {th }}$ user as $x_{k}$ and pilot $p_{k}$. The received signal can be represented as:

$$
r=\sum_{i=1}^{K} h_{k}\left(x_{k}+p_{k}\right)+n
$$

hin is the channel coefficient for $\mathbb{k}^{\text {th }}$ user.

The soft estimate of this signal $x_{k}$ is expressed as $\hat{x}_{k} \equiv\left[\hat{x}_{k}(1), \ldots \hat{x}_{k}(j), \ldots . \hat{x}_{K}(j)\right]$. Hence, the soft estimate $\hat{h}_{k}$ can be generated as:

$$
\widehat{h}_{k}=\frac{\left(\widehat{x}_{k}+p_{k}\right)^{H}\left(r-\sum_{k^{\prime} \neq k} \widetilde{h}_{k-n}\left(\widehat{x}_{k^{\prime}}+p_{k^{\prime}}\right)\right)}{\left(\widehat{x}_{k}+p_{k}\right)^{H}\left(\widehat{x}_{k}+p_{k}\right)}
$$

The subtraction method denotes the soft-cancellation method and denominator represents the data normalization. In order to improve the system performance we introduce a novel iterative process where $\hat{x}_{k}$ and $\hat{h}_{k}$ are initialized from 0 for each user $k$. With the help of Eq. (12), the receiver model initializes the channel estimating process. According to this process, the ESE and DEC functions are applied by considering $\left\{\hat{h}_{k}\right\}$ as the current channel information. The outcome of these two functions is used for updating the $\hat{x}_{k}$ by computing the mean value of the DEC outcome. The signal updating process is defined as:

$$
\widehat{x}_{k}(j)=\tanh \left\{\frac{1}{2} l_{D E C}\left(x_{k}(j)\right)\right\} \quad \forall k_{j} j
$$

Where $l_{D E C}$ is expressed as:

$$
l_{D E C}\left(x_{k}(j)\right) \equiv e_{E S E}\left(x_{k}(j)\right)+e_{D E C}\left(x_{k}(j)\right), \quad \forall k_{j} j
$$

$l_{\text {DEC }}\left(x_{k}(j)\right)$ is a posteriori information about the signal which is the outcome of DEC function. Later, the channel estimator estimates the coefficients of the channel and iterates until the entire process is finished.

Thus, we obtain the combined model of multiuser detection and channel estimation to improve the performance of IDMA system. The proposed architecture can be incorporated with MIMO-OFDM systems.

\section{RESULTS AND DISCUSSION}

In this section, we present the experimental analysis of proposed approach and compared the performance with existing techniques. The complete simulation study is carried out using MATLAB simulation tool running on windows platform with 8GB RAM and i5 64 bit processor. Complete simulation parameters are presented in below given table 1 .

\begin{tabular}{|c|c|}
\hline Parameter name & Considered Value \\
\hline $\begin{array}{c}\text { Number of } \\
\text { Subcarrier }\end{array}$ & 64 \\
\hline Modulation & QPSK \\
\hline Fading & Rayleigh \\
\hline Path Length & 16 \\
\hline FFT size & 128 \\
\hline Cyclic Prefix & 32 \\
\hline
\end{tabular}

The performance of proposed model is measured in terms of BER (Bit Error Rate) for both coded and Uncoded IDMA scenarios along with Gaussian and Non-Gaussian noise considerations. Similarly, we have measured the performance with different interleaving methods such as tree, power and random interleaving methods. Later, we have measured the performance for varied number of users. Figure 3 shows a comparative study in terms of BER vs. SNR where we compare the performance of proposed model with coded IDMA Gaussian, coded IDMA non-Gaussian, Uncoded IDMA Gaussian and Uncoded IDMA non-Gaussian.

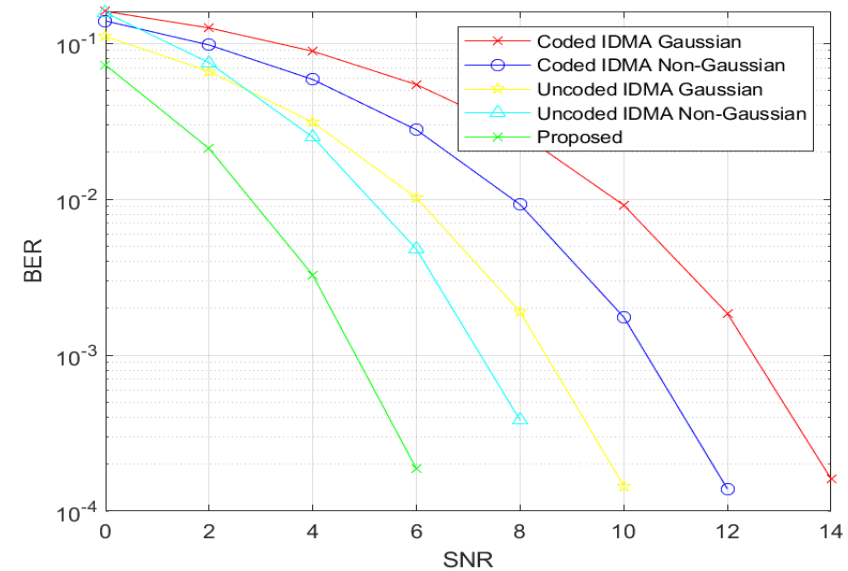

Fig.3. BER vs. SNR comparison for coded, Uncoded IDMA with Gaussian and Non-Gaussian noise.

According to figure 3, we obtain improved performance in (1 terms of BER. The proposed model is based on the coding scheme to minimize the error. This comparative study is adopted from [12]. The obtained BER values are presented in table 1 . 
Table 1 BER vs. SNR performance for coded and Uncoded IDMA

Table 1 BER vs. SNR performance for coded and Uncoded IDMA

\begin{tabular}{|c|c|c|c|c|}
\hline $\begin{array}{c}\text { Coded } \\
\text { Gaussian }\end{array}$ & $\begin{array}{c}\text { Coded-Non- } \\
\text { Gaussian }\end{array}$ & $\begin{array}{c}\text { Uncoded- } \\
\text { Gaussian }\end{array}$ & $\begin{array}{c}\text { Uncoded-Non } \\
\text { Gaussian }\end{array}$ & $\begin{array}{c}\text { Proposed } \\
\text { IDMA }\end{array}$ \\
\hline 0.160257 & 0.13916 & 0.110689 & 0.157299 & 0.072607 \\
\hline 0.125519 & 0.097559 & 0.066127 & 0.075012 & 0.021114 \\
\hline 0.088994 & 0.058618 & 0.031167 & 0.025002 & 0.00326 \\
\hline 0.054386 & 0.027871 & 0.010202 & 0.004777 & 0.000186 \\
\hline 0.026537 & 0.009247 & 0.001895 & 0.000382 & 0 \\
\hline 0.009163 & 0.001754 & 0.000145 & 0 & 0 \\
\hline 0.00185 & 0.000139 & 0 & 0 & 0 \\
\hline 0.000161 & 0 & 0 & 0 & 0 \\
\hline
\end{tabular}

This comparative study shows that the average BER performance is obtained as $0.058,0.041,0.027,0.032$ and 0.012 using Coded Gaussian, Coded-Non-Gaussian, Uncoded-Gaussian, Uncoded-Non Gaussian and Proposed IDMA, respectively. The proposed model follows iterative process which helps to reduce the bit error rates whereas conventional IDMA systems are based on the CBC detector which fails to achieve the desired performance. The proposed model shows performance improvement by $20.81 \%$,

$29.06 \%, 44.12 \%$ and 37.02 when compared with Coded Gaussian Coded-Non-Gaussian, Uncoded-Gaussian, and Uncoded-Non Gaussian, respectively.

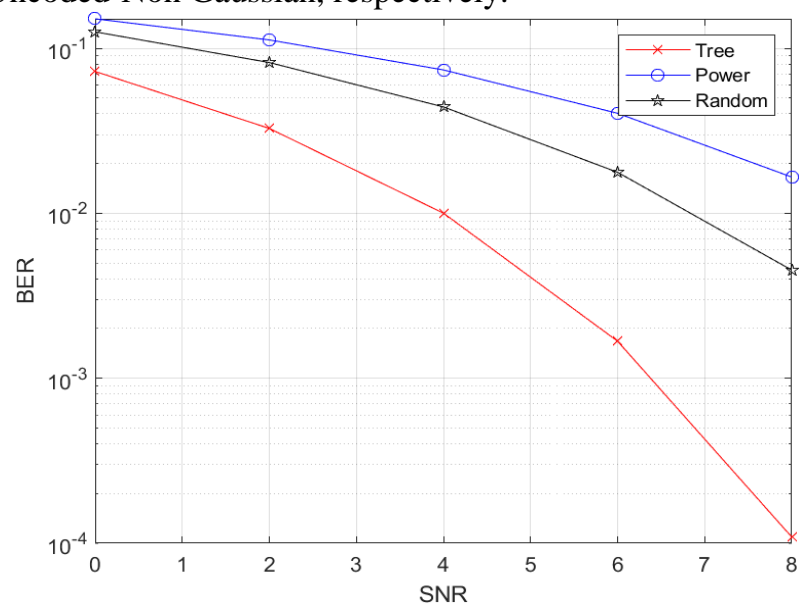

Fig.4. BER vs. SNR for different interleaving methods. Figure 4 shows a performance comparison for different types of interleaver implementation such as tree, power and random interleaving. The comparative study shows that the tree interleaving method achieves better performance due to iterative solving the channel estimation problem. The obtained BER values are presented in table 2 .

Table 2 BER vs. SNR for different Interleaving Methods

\begin{tabular}{|c|c|c|}
\hline Tree & Power & Random \\
\hline 0.072311 & 0.150755 & 0.125674 \\
\hline 0.032659 & 0.112293 & 0.081914 \\
\hline 0.010007 & 0.07389 & 0.044085 \\
\hline 0.001677 & 0.040344 & 0.017705 \\
\hline 0.000109 & 0.016565 & 0.004522 \\
\hline
\end{tabular}

The average BER is obtained as $0.023,0.078$, and 0.054 using Tree, Power and Random interleaving techniques. This comparative study shows that the proposed architecture with tree interleaving can achieve better performance. Thus, the performance of OFDM-IDMA system can be improved.

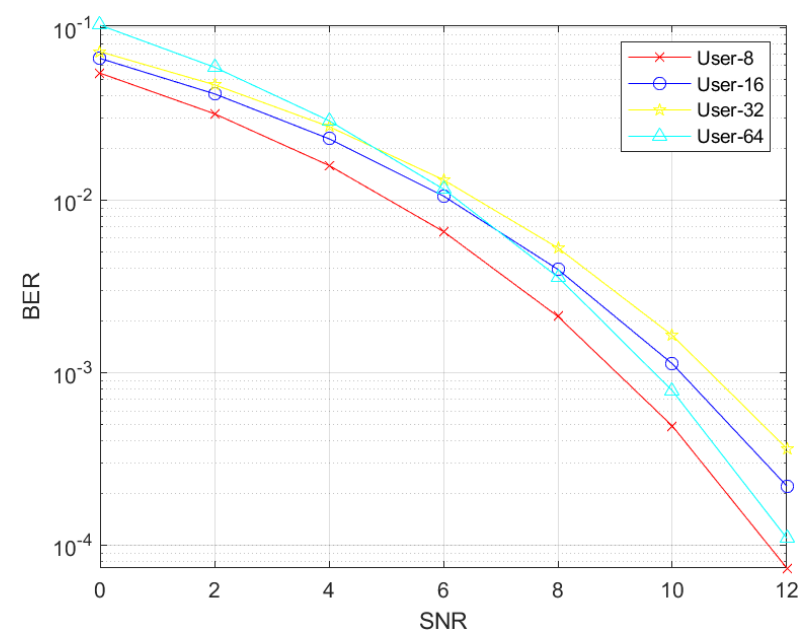

Fig.5. BER vs. SNR for varied number of users

7In another, experiment we measured the performance by considering varied number of users $K$ as $8,16,32$, and 64 . Above given figure 5 shows a comparative study for this experiment. As the numbers of users are increasing, the interference increases and channel estimation also achieves poor performance. The obtained BER values are presented in below given table 3 .

Table 3 BER vs. SNR for varied number of users

\begin{tabular}{|c|c|c|c|}
\hline 8-Users & $16-$ User & 32-Users & $64-U s e r$ \\
\hline 0.054303 & 0.066111 & 0.072311 & 0.103008 \\
\hline 0.03158 & 0.041203 & 0.046521 & 0.058803 \\
\hline 0.015849 & 0.022629 & 0.026624 & 0.028808 \\
\hline 0.006564 & 0.010541 & 0.013087 & 0.01156 \\
\hline 0.002114 & 0.00396 & 0.005274 & 0.003573 \\
\hline 0.00049 & 0.001123 & 0.001639 & 0.000784 \\
\hline $7.36 \mathrm{E}-05$ & 0.00022 & 0.000362 & 0.00011 \\
\hline
\end{tabular}

With the help of this experiment, we identify that the average BER is obtained as 0.015, 0.020, 0.023, and 0.029 for 8, 16,32 , and 64 , respectively.

\section{CONCLUSION}

In this work, we have focused on mitigating the effect of interference due to multiple access and channel estimation to improve the system performance. In order to achieve this, we consider the IDMA as base model and presented a joint approach for multiuser detection and channel estimation. The multiuser detection scheme is based on the LLR computation where mean and variance of Gaussian distribution is used for MUD. In the next phase, we present the channel estimation process where we apply Rake based soft estimation technique. 


\section{A Novel Multiuser Detection and Channel Estimation Method for Uplink Communication in MIMO-OFDM IDMA System}

Final outcome is obtained with the help of ESE and DEC of the IDMA. The performance of proposed approach is compared with different simulation parameters to prove the robustness of proposed model.

\section{REFERENCES}

1. Bilim, M., Kapucu, N., \& Develi, I. (2017). Cooperative IDMA systems with regenerative relays over Weibull fading channels: outage probability and error analysis. IET Communications, 11(7), 993-999. doi:10.1049/iet-com.2016.1322

2. https://support.chinavasion.com/index.php?/Knowledgebase/Article/ View/284/42/1g-2g-3g-4g---the-evolution-of-wireless-generations

3. Faruque, S., 2019. Time Division Multiple Access (TDMA). In Radio Frequency Multiple Access Techniques Made Easy (pp. 35-43). Springer, Cham.

4. Faruque, S., 2019. Frequency Division Multiple Access (FDMA). In Radio Frequency Multiple Access Techniques Made Easy (pp. 21-33). Springer, Cham.

5. Faruque, S., 2019. Code Division Multiple Access (CDMA). In Radio Frequency Multiple Access Techniques Made Easy (pp. 45-62). Springer, Cham

6. Vaezi, M., Ding, Z. and Poor, H.V., 2018. Multiple Access Techniques for $5 \mathrm{G}$ Wireless Networks and Beyond.

7. Wang, Q., Zhang, R., Yang, L. L., \& Hanzo, L. (2018). Non-orthogonal multiple access: A unified perspective. IEEE Wireless Communications, 25(2), 10-16.

8. Ping, L., Wang, P., \& Wang, X. (2007). Recent progress in interleave-division multiple-access (IDMA), In Invited paper, Military communication conference (MILCOM), Orlando, Florida, USA, October, pp. 29-31

9. Han, H., Li, Y., \& Xudong, G. (2019). User Identity-Aided Pilot Access Scheme for Massive MIMO-IDMA System. IEEE Transactions on Vehicular Technology.

10. Hu, Y., Xu, C., \& Ping, L. (2018, June). NOMA and IDMA in random access systems-invited paper. In 2018 IEEE 87th Vehicular Technology Conference (VTC Spring) (pp. 1-5). IEEE.

11. Sharma, D., Singh, R., \& Katiyar, H. (2019). SLLR-MDIC: Spatial log-likelihood multiuser detection and interference cancelation scheme for OFDM-IDMA. International Journal of Communication Systems, e4119.

12. Vuong, B. Q., \& Do, H. N. (2018, November). Implementation of IDMA System with Multiple Access Channel and non-Gaussian Noise. In 2018 5th NAFOSTED Conference on Information and Computer Science (NICS) (pp. 119-123). IEEE.

13. Bilim, M., Kapucu, N., \& Develi, I. (2016). A Closed-Form Approximate BEP Expression for Cooperative IDMA Systems over Multipath Nakagami- $m$ Fading Channels. IEEE Communications Letters, 20(8), 1599-1602.

14. Li, B., Du, R., Kang, W., \& Liu, G. (2017). Multi-user detection for sporadic IDMA transmission based on compressed sensing. Entropy, 19(7), 334.

15. Meng, X., Wu, S., Kuang, L., Ni, Z., \& Lu, J. (2014, May). Expectation propagation based iterative multi-user detection for MIMO-IDMA systems. In 2014 IEEE 79th Vehicular Technology Conference (VTC Spring) (pp. 1-5). IEEE.

16. Chen, M., \& Burr, A. G. (2013, March). Low-Complexity Iterative Interference Cancellation Multiuser Detection for Overloaded MIMO OFDM IDMA System. In WSA 2013; 17th International ITG Workshop on Smart Antennas (pp. 1-5). VDE.

17. Botsinis, P., Alanis, D., Babar, Z., Ng, S. X., \& Hanzo, L. (2015). Iterative quantum-assisted multi-user detection for multi-carrier interleave division multiple access systems. IEEE Transactions on Communications, 63(10), 3713-3727.

18. Oyerinde, O. O. (2018). An overview of channel estimation schemes based on regularized adaptive algorithms for OFDM-IDMA systems. Digital Signal Processing, 75, 168-183.

19. Chang, C. F., Liu, Y. C., Yu, M. H., \& Lin, M. C. (2016). A joint design of timing tracking and channel estimation for IDMA receivers. In 2016 International Symposium on Information Theory and Its Applications (ISITA) (pp. 186-190). IEEE.

20. Rizaner, A. (2017). A subspace channel estimation method for IDMA in $5 \mathrm{G}$ systems. Optik, 148, 251-255.

21. Bilim, M., Kapucu, N., \& Develi, I. (2018). Application of best relay selection approach to cooperative DF IDMA systems over Rayleigh/Weibull fading channels. International Journal of Communication Systems, 31(5), e3506.

22. Shukla, A., Goyal, V., Mishra, P., \& Deolia, V. K. (2018). Cooperative relay beamforming in IDMA communication networks. Journal of Electrical Engineering, 69(4), 300-304.

23. Vishvaksenan, K. S., Rajmohan, R., \& Kalaiarasan, R. (2017, April) Multi-carrier IDMA system for relay aided cooperative downlink communication with transmitter preprocessing. In 2017 International Conference on Communication and Signal Processing (ICCSP) (pp 2206-2210). IEEE.

24. Shikida, J., Suyama, S., Suzuki, H., \& Fukawa, K. (2010, May). Iterative receiver employing multiuser detection and channel estimation for MIMO-OFDM IDMA. In 2010 IEEE 71st Vehicular Technology Conference (pp. 1-5). IEEE.

25. Taşpinar N., \& Şimşir, Ş. (2014, September). Channel estimation techniques in OFDM-IDMA systems. In 2014 2nd International Symposium on Wireless Systems within the Conferences on Intelligent Data Acquisition and Advanced Computing Systems (pp. 10-14) IEEE.

26. Peng, T., Xiao, Y., He, X., \& Li, S. (2012). Improved detection of uplink OFDM-IDMA signals with carrier frequency offsets. IEEE Communications Letters, 16(5), 646-649.

27. Chen, M., \& Burr, A. G. (2013, March). Low-Complexity Iterative Interference Cancellation Multiuser Detection for Overloaded MIMO OFDM IDMA System. In WSA 2013; 17th International ITG Workshop on Smart Antennas (pp. 1-5). VDE.

28. Zhou, X., Zhang, H., \& Yuan, D. (2010, December). A multi-source cooperative scheme based on IDMA aided superposition modulation. In 2010 IEEE International Conference on Information Theory and Information Security (pp. 802-806). IEEE.

29. Yang, Y. D., Zhou, X. L., \& Hu, D. (2011). Performance analysis of network coding relay cooperative IDMA system with Turbo code in multi-path channel. Information and Electronic Engineering, 9(2), 143-147.

30. Zhang, J. (2019, June). Performance Enhancement of IDMA System by Power and LDPC Code Optimization. In 2019 International Conference on Wireless Communication, Network and Multimedia Engineering (WCNME 2019). Atlantis Press.

31. Zhang, Y., Peng, K., \& Song, J. (2017, December). Enhanced IDMA with rate-compatible raptor-like quasi-cyclic LDPC code for 5G. In 2017 IEEE Globecom Workshops (GC Wkshps) (pp. 1-6). IEEE. 